\title{
Cancer and radiotherapy-related fatigue: implications and therapies integratives.
}

Clevia Santos Passos ${ }^{1 *}$, Erica Regina Ribeiro Sady ${ }^{3}$, Rodrigo Vanerson Passos Neves ${ }^{2}$, Bruna Elisa Piovezani Carvalho ${ }^{1}$, Tassia Santos Rodrigues Costa ${ }^{1}$, Anderson Sola Haro ${ }^{4}$, Andreia Gonçalves Silva $^{4}$, Fernando Costa ${ }^{4}$, Thiago Santos Rosa ${ }^{2}$, Adriana Sarmento Oliveira ${ }^{1}$

${ }^{1}$ Faculdade de Medicina FMUSP, Universidade de Sao Paulo, Sao Paulo, SP, Brazil

${ }^{2}$ Graduate Program in Physical Education, Universidade Catolica de Brasilia, Taguatinga, DF, Brazil

${ }^{3}$ Cardiovascular Physiotherapist at the Hospital Beneficência Portuguesa in Sao Paulo, Sao Paulo, SP, Brazil

${ }^{4}$ Independent Researcher, São Paulo, SP, Brazil

\begin{abstract}
Radiation therapy is a strategy widely used to treat or prevent the onset of tumors located in a particular region of the body, however, depending on the body area affected by cancer, the prescribed dose of ionizing radiation, and the technique used, they may interfere in the beneficial or side effects. Fatigue has been one of the most persistent effect before, during or after cancer treatment, whether caused by the type of treatment, as surgery, chemotherapy and radiation therapy, this may be combined or used alone. Objective was to review the fatigue related to radiotherapy and cancer, as well as the implications and therapeutics used in the treatment of cancer. The search for scientific articles was in PubMed and Scielo database. Our results show that metabolic, nutritional and adaptive changes to treatment and the own cancer affect the therapeutic follow-up and quality of life, but the inclusion of alternative therapies such as acupuncture and physical exercise have attenuated undesirable symptoms. Thus, it is suggested the inclusion of a multidisciplinary approach to cancer treatment. The well targeted cancer treatment will provide the reduction of possible adverse sideeffects and with attenuation of fatigue thus favor better quality of life for patients.
\end{abstract}

Keywords: Cancer, Fatigue, Exercise, Acupuncture, Radiation therapy, Quality of life.

Accepted on 20 March 2018

\section{Introduction}

The cancer is considered a chronic disease and a major cause of death worldwide and is a serious public health problem. Studies have shown a tendency of exponential growth of this disease in the coming decades. The World Health Organization (WHO) estimates that by the year 2030, 75 million people will be affected by cancer, 27 million incidents cases and 17 million deaths by cancer in the world [1] .

Radiotherapy (RT) is one of the treatment options that can be used alone or adjuvant in cancer [2]. Combinations are varied, can be used before, during or after chemotherapy or surgery. In addition, it may be indicated for prophylaxis, reduction of tumor volume, total cure or to avoid remission. However, fatigue is one of the most prevalent and distressing symptoms reported by cancer patients [3].

Cancer-related fatigue (CRF) is defined by National Comprehensive Cancer Network (NCCN) as "a distressing, persistent, subjective sense of physical fatigue, emotional and / or cognitive tiredness or exhaustion related to cancer or cancer treatment that is not proportional to the recent activity and interferes with usual functioning" [4]. Affects a significant number of individuals diagnosed with cancer and according to population, type of treatment and evaluation method, the ratio of affected CRF ranging from $25 \%$ to $99 \%$. Of these $30 \%$ to $60 \%$, the fatigue is presented moderate to high during the treatment of cancer, although disappears after one year of the end of treatment, there are a portion of the population (1/4) who feel the symptoms of fatigue longer, which comes to overcome the ten years [5].

As a form of treatment for CRF there are two main approaches, pharmacological and other non-pharmacological [1]. Within the pharmacological line there is an attempt to control factors such as elevation of inflammatory cytokines, diabetes mellitus and anemia with positive results. [4]. Also in this line of treatment the use of corticosteroids [6], The psychotropic methylphenidate, both with positive results; On the other side modafinil did not show positive effects in the treatment of symptoms, with results similar to those presented by the control group [6,7]. The second approach is the non-pharmacological treatment, and exercise focused studies to combat fatigue both in adults and in young [8] with positive results in the field [9].

Pathophysiological mechanisms of CRF are not fully known. However, it is proposed that some mechanisms are related to inflammatory aspects; Imbalance of the hypothalamicpituitary-adrenal axis; Desynchronization of the circadian rhythm; Musculoskeletal disorder and genetic dysregulation [10-12]. 
Citation: Passos CS, Sady ERR, Neves RVP, et al. Cancer and radiotherapy-related fatigue: implications and therapies integratives. J Med Oncl Ther 2018;3(1):9-14.

A disease related to these features, is obesity. This has been considered a public health problem[13]. Excessive accumulation can increase levels of inflammation and hormonal changes associated with the development of cancer $[13,14]$. In addition, overweight and obese patients tend to receive a higher dose of radiotherapy and thereby increase inflammatory levels and CRF. The dose of radiation administered in obese patients may increase the risk of skin burns, as well as increases the chance of cancer recurrence, as well as quality of life is adversely affected during and after treatment [15].

A study of endometrial cancer, showed data that young morbid obese women have an increased risk of toxicity compared to other population. Because the population is expected to avoid adverse side effects of the doses given for tumor control [16]. Therefore, it is necessary to determine methods and techniques of radiotherapy to have correct delivery and lower toxicity results in these patients [17]. Moreover, additional research focusing on quality of life, fatigue by radiotherapy, and problems associated with obesity, medications and comorbidities, and adjunctive therapies are needed to reduce chronic inflammation and abnormal release of cytokines that may contribute to obesity and cancer [14].

Several studies suggest a significant association between inflammation and cancer, wherein chronic inflammation is shown as an epigenetic factors that corroborate the formation and tumor course. According to tumor location and individual factors of the host, inflammation associated with cancer and their role in neoplastic progression are dynamic and variable [18].

Thus, evidence suggests that tumor cells are capable of autoregular the expression of cytokines, chemokines and other mediators to facilitate the recruitment of circulating immune cells and subsequently to contribute to neoplastic progression [19]. In the case of radiotherapy, studies show that radiation may involve the treatment of cancer patients, suggesting the importance of identifying inflammatory mechanisms as potential mediators of radiation-induced fatigue, Since peripheral-induced pro-inflammatory cytokines may produce changes in neural activity in the face of persistent cytokine exposure in the central nervous system which could explain the inflammatory processes in cancer patients and fatigue-related symptoms [20]. In the case of severe fatigue, the inclusion of systemic markers of inflammatory activity may indicate changes following treatment, as the quality of life of patients undergoing radiation oncology may be affected during and after treatment $[19,20]$.

Amongst other cancer events, especially in the advanced stage, there is a reduction in the appetite and alteration of the patient's metabolism associated with the release of pro-inflammatory factors by the host and other catabolic factors generated by the tumor, leading to anorexia-cachexia syndrome (ACS), observed by response to chronic inflammation, depletion of essential amino acids and negative nitrogen balance and lipolysis [21,22]. The immune system is capable of modulating the expression of damage induced by radiation and radiationinduced tumor tissue [2], that is, it can influence the cure of the disease, as stimulating side effect of the short or long term radiotherapy.

Thus, it may increase the effect of fatigue, which may be primary when triggered by cancer in response to persistent proinflammatory cytokines, as well as secondary to medications and comorbidities such as anemia, cachexia, pain, infections, metabolic and endocrine disorders and sedentary lifestyle. ACS is intensified by changes in nutrient metabolism, hormonal changes, and increased circulating cytokines. Changes in the perception of taste and smell occurred with tumor progression and cancer treatment also contributes to anorexia, and consequently with the ACS [23].

However, considering the CRF a subjective symptom, it can be assessed by self report instruments, among others. Moreover, CRF should be recognized, measured, documented and managed according to clinical practice guidelines, among all age groups and for all stages of cancer disease, the initial queries and at regular intervals (during and after cancer treatment including the terminal illness period), and when clinically indicated [24].

In addition to detailed CRF investigation (onset, duration, temporal variation, worsening and relief factors, functional impact), appropriate evaluation should also consider cancer diagnosis, disease status (recurrence and progression), and related treatments (pharmacological and nonpharmacological). Rarely isolated, the CRF is associated with other symptoms multiple - which vary depending on the diagnosis, treatment and disease stage [24].

For this reason, it should be performed extensive review of systems; Sizing of social support and economic status of the individual; as well as research potentially treatable factors such as pain; emotional distress (anxiety and depression); anemia; sleep disorders; nutritional deficits; functional status; pharmacological interactions and side effects of drugs; and comorbidities [24].

Interventions are based on educational counseling of individuals with cancer disease and their families, with strategies to identify and monitor the CRF; adequacy of expectations and establishing priorities, through adjustments in the pace of life, activity schedules, delegation of tasks and postponement of those non-essential; energy conservation techniques, through the use of assistive devices [24- 26].

Furthermore, psychological support, through cognitive behavioral therapy relaxation strategies, guidance on naps $<1$ hour and "hygiene" of the environment not to interfere with the quality of nighttime sleep; Educational and support psychotherapy (group therapy, counseling, orientation on leisure activities and use of distractors, maintenance of social support), and nutritional, with adequate hydration and electrolyte balance, are fundamental [24-26].

\section{Therapy of Fatigue in Cancer}

Quality of life is a thoroughly studied concept that integrates different areas of knowledge. This concept has been applied systematically since the 70 's in the health Field [27]. A focus 
on quality of life is the importance invested in prevention, treatment and rehabilitation, thus needing to verify the subjective perceptions of patients with chronic diseases. However, the concept does not always include which often refers to the term as a synonym for improving the health status of the patient [28].

The choice of treatment can influence the response of quality of life and health of the patient with cancer. However, the advanced stage requires more severe clinical measures to extend life expectancy [29].The need to evaluate the health condition of the patients, aims to optimize resources for the different cultural aspects, influencing the therapeutic decision. The multidisciplinary approach of different teams on a subject as complex as the subjective perception of health during and after treatment, has been an effective output in fighting cancer [30].

Acupuncture is one of the complementary therapies that have been used to increase the immune system and alleviate the side effects, increasing the tolerability to conventional cancer treatment [31-32]. Acupuncture is an ancient science which aims to promote circulation and energy release aimed at healing and / or prevention of diseases and the overall balance in the body by applying stimuli through the skin by inserting needles at specific points, called acupoints [33]. Some of the mechanisms of action after insertion of the needle and the generation of "Qi" is the release of beta-endorphins in the spine and supraspinatus, neuropeptides proinflammatory and increased peripheral circulation. [34-36].

There is evidence that acupuncture can be an alternative in reducing cancer-related symptoms commonly seen in patients undergoing radiation therapy. Studies have shown that acupuncture reduces fatigue [37], nausea [38], xerostomia [39], pain [40], dysphagia [41] and anorexia [42], and increase feeding capacity [43] and quality of life [39] of cancer patients undergoing to radiotherapy.

All studies were randomized, placebo controlled group. Specifically with respect to fatigue, acupuncture's performance in all the other symptoms mentioned above, by itself, softens it. In the study by Balk et al [37], Acupuncture was performed once or twice a week for four weeks. however, additional studies are needed to explain the mechanisms responsible for these effects $[44,45]$.

Although these studies scientifically evidence the possible benefits of acupuncture to minimize the adverse effects of the treatment in several types of cancer with radiotherapy in patients, [46], Mainly in relation to fatigue, acupuncture has been used by only $5 \%$ to $10 \%$ of cancer patients [47]. Possibly, according to studies with systematic reviews and metaanalyzes on various symptoms of cancer, interventions are still heterogeneous. [44,45] .

The World Health Organization [48] emphasizes the equal importance in promoting palliative care for cancer patients, being one of the four integrative treatment planning the key posts of the World Health Organization for cancer patients.
Since cancer is associated with the decline of vital functions, and thus the loss of lean mass, which tends to worsen with progression of the disease, affecting the quality of life and reducing the efficiency of medical treatment [49], research has been developed with application of physical exercise, acting in concomitance to the treatment in the improvement of functional parameters of the metabolism that integrate part of the quality of life [50].

Thus, as a treatment strategy, physical exercise has shown positive results for health. The assessment of well-being, achieved through quality of life questionnaires shows that even in individuals affected by an advanced stage of cancer, submit it to resistance exercise can be interesting and efficient for the maintenance of lean body mass, corroborating health parameters and quality of life of affected patients with cancer[51,52].

\section{Physical Exercise, Cancer and Fatigue}

Cancer treatments are used for the purpose of curing or even prolonging the lives of these patients [53]. One of the modalities for the treatment of cancer is the radiotherapy, which is commonly used in the attempt to attack the cancerous cells and at the same time end up also affecting healthy cells [54].

However, treatment with radiation therapy induces some adverse effects, such as fatigue, which negatively affect the functional capacity and quality of life of these people [55]. Radiation-related fatigue is a serious clinical problem, and its cause is multifactorial, in which several mechanisms have been proposed, including genetic, inflammatory, immunological, psychological, hormonal factors and hemoglobin levels [3,56-60]. All of these etiological factors in fatigue related to radiotherapy play relevant roles in impairment of oxygen delivery, weight loss and apetite [61] and neuromuscular fatigue [62]. As well as, low levels of physical activity, mass and muscle strength can cooperate for fatigue [63].

On the other side, chronically aerobic and strength exercise physical is an interesting tool in combating physical fatigue, increases cardiorespiratory fitness and muscle strength in patients treated with breast cancer [64]. Physical exercise plays an important role in the etiological factors involved in fatigueIt is known that physical exercise chronically improves inflammatory, immunological, cardiovascular, anthropometric parameters in people with cancer [65-69], and there seems to be a direct relationship with adherence to physical exercise [66].

In addition, it has been reported that these patients have low levels of cardiorespiratory fitness and muscular strength, which partially explains fatigue related to radiotherapy [70]. Therefore, combined exercise training (aerobic + strength) can be used to improve oxygen consumption (VO2), reduce body adiposity, increase mass and strength muscular [68,71].

In this sense, the association of aerobic training with strength training is an interesting tool that, through action in several physiological pathways, attenuates the disorders caused by fatigue related to radiotherapy, improving the quality of life of 
Citation: Passos CS, Sady ERR, Neves RVP, et al. Cancer and radiotherapy-related fatigue: implications and therapies integratives. J Med Oncl Ther 2018;3(1):9-14.

these patients [69]. However, future studies with the objective of evaluating other modalities of exercise, intensity, duration are necessary, as well as the influence of physical exercise on fatigue related to radiotherapy of several types of cancers.

\section{Final Considerations}

Given the evident economic impact of disability related to CRF, diagnosed individuals should be promptly referred to specialists in physical rehabilitation, such as physical educators, physiatrists, physiotherapists and occupational therapists, aiming to maintain an optimal functional level [24]. Therefore, an individualized exercise program should be established, combining aerobic and resistance activities, together with those that promote relaxation (acupuncture; yoga, massage) [72-74].

In addition, the pharmacological aspect, medical interventions aimed at CRF should consider prescribing psychostimulants and treatment of pain, emotional distress; anemia and optimize the treatment of sleep disorders, nutritional and associated comorbidities. However, it is essential that Health Professional, consider aspects related to metastases, especially bone; thrombocytopenia; neutropenia; anemia; fever; active infection [24].

Thus, is suggested that the joint action of multidisciplinary facilitate the association of various pharmacological and nonpharmacological interventions as adjuvants in the impact of CRF, which will be positive during and after cancer treatment, allowing improving the quality of life of cancer patients.

\section{References}

1. WHO. International Agency for Research on Cancer. World Health Organization- World Cancer Report 2008. Lyon:IARC Press. 2008.

2. Schaue D, Micewicz ED, Ratikan JA, et al. Radiation and inflammation. Semin Radiat Oncol. 2015;25:4-10.

3. Wang XS. Pathophysiology of cancer-related fatigue. Clin J Oncol Nurs. 2008;12:11-20.

4. Berger AM, Mooney K, Alvarez-Perez A, et al. Cancerrelated fatigue, version 2.2015. J Natl Compr Canc Netw. 2015;13:1012-39.

5. Servaes P, Verhagen C, Bleijenberg G. Fatigue in cancer patients during and after treatment: Prevalence, correlates and interventions. Eur J Cancer Care. 2002;38:27-43.

6. Begley S, Rose K, O'Connor M. The use of corticosteroids in reducing cancer-related fatigue: assessing the evidence for clinical practice. Int J Palliat Nurs. 2016;22:5-9.

7. Qu D, Zhang Z, Yu X, et al. Psychotropic drugs for the management of cancerrelated fatigue: A systematic review and metaanalysis. Eur J Cancer Care. 2016;25:970-9.

8. LopesJúnior L, Bomfim E, Nascimento L, et al. Nonpharmacological interventions to manage fatigue and psychological stress in children and adolescents with cancer: An integrative review. Eur J Cancer Care. 2016;25:921-35.
9. Scott K, Posmontier B. Exercise interventions to reduce cancer-related fatigue and improve health-related quality of life in cancer patients. Holist Nurs Pract. 2017;31:66-79.

10. Berger AM, Wielgus K, Hertzog M, et al. Patterns of circadian activity rhythms and their relationships with fatigue and anxiety/depression in women treated with breast cancer adjuvant chemotherapy. Support Care Cancer. 2010;18:105-14.

11. Schubert C, Hong S, Natarajan L, et al. The association between fatigue and inflammatory marker levels in cancer patients: A quantitative review. Brain Behav Immun. 2007;21:413-27.

12. al-Majid S, McCarthy DO. Cancer-induced fatigue and skeletal muscle wasting: The role of exercise. Biol Res Nurs. 2001;2:186-97.

13. Passos CS RR, Rosa TS, Neves RVP, et al. Obesity, inflammation, physical inactivity and risk for cancer. $\mathrm{J}$ Med Oncl Ther. 2017;2:16-9.

14. Tao W, Lagergren J. Clinical management of obese patients with cancer. Nat Rev Clin Oncol. 2013;10:519-33.

15. Fader AN, Frasure HE, Gil KM, et al. Quality of life in endometrial cancer survivors: What does obesity have to do with it? Obstet Gynecol Int. 2011;2011:308609.

16. Dandapani SV, Zhang Y, Jennelle R, et al. Radiationassociated toxicities in obese women with endometrial cancer: More than just BMI? Sci World J. 2015:483208.

17. Smyth H . Does obesity impact treatment outcome for prostate cancer patients treated with radiotherapy: The weighted debate. J Cancer Prev Curr Res. 2015;2:21.

18. Balkwill F, Mantovani A. Inflammation and cancer: Back to Virchow? Lancet. 2001;357:539-45.

19. Schreiber RD, Old LJ, Smyth MJ. Cancer immunoediting: Integrating immunity's roles in cancer suppression and promotion. Science. 2011;331:1565-70.

20. Bower JE, Ganz PA, Tao ML,et al. Inflammatory biomarkers and fatigue during radiation therapy for breast and prostate cancer. Clin Cancer Res. 2009;1:5534-40.

21. Tisdale MJ. The 'cancer cachectic factor'. Support Care Cancer. 2003;11:73-8.

22. Tisdale MJ. Cachexia in cancer patients. Nat Rev Cancer. 2002;2:862-71.

23. Inui A. Cancer anorexia-cachexia syndrome: Current issues in research and management. CA Cancer J Clin. 2002;52:72-91.

24. NCCN. Cancer-Related Fatigue: In: Network N, ed. NCCN Clinical Practice Guidelines in Oncology (NCCN Guidelines). 2017.

25. Pachman DR, Price KA, Carey EC. Nonpharmacologic approach to fatigue in patients with cancer. Cancer J. 2014;20:313-8.

26. Given BA, Given CW, Kozachik S. Family support in advanced cancer. CA Cancer J Clin. 2001;51:213-31.

27. Mansano-Schlosser TC, Ceolim MF. Qualidade de vida de pacientes com câncer no período de quimioterapia. Texto \& Contexto - Enfermagem. 2012;21:600-7. 
28. Saad IA, Botega NJ, Toro IF. Predictors of quality-of-life improvement following pulmonary resection due to lung cancer. Sao Paulo Med J 2007;125:46-9.

29. Jayadevappa R, Chhatre S, Wong YN, et al. Comparative effectiveness of prostate cancer treatments for patientcentered outcomes: A systematic review and meta-analysis (PRISMA Compliant). Medicine (Baltimore) 2017;96:6790.

30. Sawada NO, Nicolussi AC, Okino L, et al. Quality of life evaluation in cancer patients to submitted to chemotherapy. Rev Esc Enferm USP. 2009;43:581-7.

31. Sertel S, Herrmann S, Greten HJ, et al. Additional use of acupuncture to NSAID effectively reduces posttonsillectomy pain. Eur Arch Otorhinolaryngol. 2009;266:919-25.

32. Wenzel KW. Acupuncture: what is the outcome of the Gerac Studies. Dtsch Med Wochenschr 2005;130:1520.

33. Oliveira AS, Filho JHAC, Oliveira OS, et al. Analgesic action of acupuncture. Unesciences. 2009;2:81-92.

34. Liu S, Zhou W, Ruan X, et al. Activation of the hypothalamus characterizes the response to acupuncture stimulation in heroin addicts. Neurosci Lett. 2007;421:203-8.

35. Pariente J, White P, Frackowiak RS, et al. Expectancy and belief modulate the neuronal substrates of pain treated by acupuncture. Neuroimage. 2005;25:1161-7.

36. Carlsson C. Acupuncture mechanisms for clinically relevant long-term effects--reconsideration and a hypothesis. Acupunct Med. 2002;20:82-99.

37. Balk J, Day R, Rosenzweig M, et al. Pilot, randomized, modified, double-blind, placebo-controlled trial of acupuncture for cancer-related fatigue. J Soc Integr Oncol. 2009; 7:4-11.

38. Enblom A, Johnsson A, Hammar M, et al. Acupuncture compared with placebo acupuncture in radiotherapyinduced nausea--a randomized controlled study. Ann Oncol 2012;23:1353-61.

39. Meng Z, Garcia MK, Hu C, et al. Randomized controlled trial of acupuncture for prevention of radiation-induced xerostomia among patients with nasopharyngeal carcinoma. Cancer. 2012;118:3337-44.

40. Pfister DG, Cassileth BR, Deng GE, et al. Acupuncture for pain and dysfunction after neck dissection: Results of a randomized controlled trial. J Clin Oncol. 2010;28:2565-70.

41. Lu W, Wayne PM, Davis RB, et al. Acupuncture for chemoradiation therapy-related dysphagia in head and neck cancer: A pilot randomized sham-controlled trial. Oncologist. 2016;21:1522-9.

42. Jeon JH, Yoon J, Cho CK, et al. Effect of acupuncture for radioactive-iodine-induced anorexia in thyroid cancer patients: a randomized, double-blinded, sham-controlled pilot study. Integr Cancer Ther. 2015;14:221-30.

43. Enblom A, Steineck G, Hammar M, et al. Reduced need for rescue antiemetics and improved capacity to eat in patients receiving acupuncture compared to patients receiving sham acupuncture or standard care during radiotherapy. Evid Based Complement Alternat Med. 2017:5806351.

44. Grant SJ, Smith CA, de Silva N, et al. Defining the quality of acupuncture: The case of acupuncture for cancer-related fatigue. Integr Cancer Ther. 2015;14:258-70.

45. Zeng Y, Luo T, Finnegan-John J, et al. Meta-analysis of randomized controlled trials of acupuncture for cancerrelated fatigue. Integr Cancer Ther. 2014;13:193-200.

46. Lian WL, Pan MQ, Zhou DH, et al. Effectiveness of acupuncture for palliative care in cancer patients: A systematic review. Chin J Integr Med. 2014;20:136-47.

47. Mao JJ, Palmer CS, Healy KE, et al. Complementary and alternative medicine use among cancer survivors: A population-based study. J Cancer Surviv. 2011;5:8-17.

48. WHO. Definition of palliative care.

49. Aversa Z, Costelli P, Muscaritoli M. Cancer-induced muscle wasting: latest findings in prevention and treatment. Ther Adv Med Oncol. 2017;9:369-82.

50. Wurz A, Brunet J. The effects of physical activity on health and quality of life in adolescent cancer survivors: A systematic review. JMIR Cancer. 2016;2:6.

51. Lonkvist CK, Lønbro S, Vinther A, et al. Progressive resistance training in head and neck cancer patients during concomitant chemoradiotherapy - design of the DAHANCA 31 randomized trial. BMC Cancer. 2017; 17:400.

52. Neefjes ECW, van den Hurk RM, Blauwhoff-Buskermolen $\mathrm{S}$, et al. Muscle mass as a target to reduce fatigue in patients with advanced cancer. J Cachexia Sarcopenia Muscle. 2017.

53. Solin LJ. Breast conservation treatment with radiation: An ongoing success story. J Clin Oncol. 2010;28:709-11.

54. Gami B, Harrington K, Blake $\mathrm{P}$, et al. How patients manage gastrointestinal symptoms after pelvic radiotherapy. Aliment Pharmacol Ther. 2003;18:987-94.

55. Noal S, Levy C, Hardouin A, et al. One-year longitudinal study of fatigue, cognitive functions, and quality of life after adjuvant radiotherapy for breast cancer. Int J Radiat Oncol Biol Phys. 2011;81:795-803.

56. Bock PR, Hanisch J, Matthes H, et al. Targeting inflammation in cancer-related-fatigue: A rationale for mistletoe therapy as supportive care in colorectal cancer patients. Inflamm Allergy Drug Targets. 2014;13:105-11.

57. Courtier N, Gambling T, Enright S, et al. Psychological and immunological characteristics of fatigued women undergoing radiotherapy for early-stage breast cancer. Support Care Cancer. 2013;21:173-81.

58. Schmidt ME, Chang-Claude J, Seibold P, et al. Determinants of long-term fatigue in breast cancer survivors: Results of a prospective patient cohort study. Psychooncology. 2015;24:40-6.

59. Saligan LN, Kim HS. A systematic review of the association between immunogenomic markers and cancerrelated fatigue. Brain Behav Immun. 2012;26:830-48.

60. Henke M, Guttenberger R, Barke A, et al. Erythropoietin for patients undergoing radiotherapy: A pilot study. Radiother Oncol. 1999;50:185-90. 
Citation: Passos CS, Sady ERR, Neves RVP, et al. Cancer and radiotherapy-related fatigue: implications and therapies integratives. J Med Oncl Ther 2018;3(1):9-14.

61. Gutstein HB. The biologic basis of fatigue. Cancer 2001;92:1678-83.

62. Monga U, Jaweed M, Kerrigan AJ, et al. Neuromuscular fatigue in prostate cancer patients undergoing radiation therapy. Arch Phys Med Rehabil. 1997;78:961-6.

63. Al-Majid S, Gray DP. A biobehavioral model for the study of exercise interventions in cancer-related fatigue. Biol Res Nurs. 2009;10:381-91.

64. Travier N, Velthuis MJ, Steins Bisschop CN, et al. Effects of an 18-week exercise programme started early during breast cancer treatment: A randomised controlled trial. BMC Med. 2015;13:121.

65. Schmidt ME, Meynkoh A, Habermann N, et al. Resistance exercise and inflammation in breast cancer patients undergoing adjuvant radiation therapy: Mediation analysis from a randomized, controlled intervention trial. Int $\mathrm{J}$ Radiat Oncol Biol Phys. 2016;94:329-37.

66. Peters C, Lotzerich H, Niemeier B, et al. Influence of a moderate exercise training on natural killer cytotoxicity and personality traits in cancer patients. Anticancer Res. 1994;14:1033-6.

67. Hojan K, Kwiatkowska-Borowczyk E, Leporowska E, et al. Physical exercise for functional capacity, blood immune function, fatigue, and quality of life in high-risk prostate cancer patients during radiotherapy: A prospective, randomized clinical study. Eur J Phys Rehabil Med. 2016;52:489-501.

68. Rahnama N, Nouri R, Rahmaninia F, et al. The effects of exercise training on maximum aerobic capacity, resting heart rate, blood pressure and anthropometric variables of postmenopausal women with breast cancer. J Res Med Sci. 2010;15:78-83.

69. Lipsett A, Barrett S, Haruna F, et al. The impact of exercise during adjuvant radiotherapy for breast cancer on fatigue and quality of life: A systematic review and metaanalysis. Breast. 2017;32:144-155.

70. Clauss D, Tjaden C, Hackert $\mathrm{T}$, et al. Cardiorespiratory fitness and muscle strength in pancreatic cancer patients. Support Care Cancer 2017.

71. Cornette T, Vincent F, Mandigout S, et al. Effects of homebased exercise training on $\mathrm{VO} 2$ in breast cancer patients under adjuvant or neoadjuvant chemotherapy (SAPA): A randomized controlled trial. Eur J Phys Rehabil Med. 2016;52:223-32.

72. Mishra SI, Scherer RW, Snyder C, et al. Exercise interventions on health-related quality of life for people with cancer during active treatment. Cochrane Database Syst Rev. 2012:08465.

73. Schmitz KH, Courneya KS, Matthews C, et al. American College of Sports Medicine roundtable on exercise guidelines for cancer survivors. Med Sci Sports Exerc. 2010;42:1409-26.

74. Tian L, Lu HJ, Lin L, et al. Effects of aerobic exercise on cancer-related fatigue: A meta-analysis of randomized controlled trials. Support Care Cancer. 2016;24:969-83.

\section{*Correspondence to:}

Clevia Santos Passos

Incor/FMUSP

Bairro Cerqueira Cesar

São Paulo - SP, Brasil

Tel: 5511995570466

E-mail: cleviasp@gmail.com 